\title{
Lyapunov functional techniques for the global stability analysis of a delayed SIRS epidemic model
}

\author{
Yoichi Enatsu $^{\mathrm{a}, *}$, Yukihiko Nakata ${ }^{\mathrm{b}}$, Yoshiaki Muroya ${ }^{\mathrm{c}}$ \\ ${ }^{a}$ Department of Pure and Applied Mathematics, Waseda University, 3-4-1 Ohkubo, \\ Shinjuku-ku, Tokyo, 169-8555, Japan \\ ${ }^{b}$ Basque Center for Applied Mathematics, Bizkaia Technology Park, Building 500 E-48160 \\ Derio, Spain \\ ${ }^{c}$ Department of Mathematics, Waseda University 3-4-1 Ohkubo, Shinjuku-ku, Tokyo \\ 169-8555, Japan
}

\begin{abstract}
In this paper, we study the global dynamics of a delayed SIRS epidemic model for transmission of disease with a class of nonlinear incidence rates of the form $\beta S(t) \int_{0}^{h} f(\tau) G(I(t-\tau)) d \tau$. Applying Lyapunov functional techniques in the recent paper [Y. Nakata, Y. Enatsu and Y. Muroya, On the global stability of an SIRS epidemic model with distributed delays, accepted], we establish sufficient conditions of the rate of immunity loss for the global asymptotic stability of an endemic equilibrium for the model. In particular, we offer a unified construction of Lyapunov functionals for both cases of $R_{0} \leq 1$ and $R_{0}>1$, where $R_{0}$ is the basic reproduction number.
\end{abstract}

Keywords: SIRS epidemic model; nonlinear incidence rate; global asymptotic stability; Lyapunov functional; distributed delays

\section{Introduction}

In order to understand epidemiological patterns and control communicable diseases, we have obtained qualitative results of stability analyses of epidemic models (see [1]-[23] and the references therein).

Mena Lorcat and Hethcote [15] formulated SIRS (Susceptible - Infected Recovered - Susceptible) epidemic models, which were initially applied to fit data for infectious diseases as regulators of laboratory population of mice.

In order to investigate the effect of the impermanent immunity of vectorborne diseases, many authors have now carried out stability analysis of the equilibria for delayed SIRS epidemic models [16, 20, 21, 22, 23].

\footnotetext{
* Corresponding author.

Email addresses: yo1.gc-rw.docomo@akane.waseda.jp (Yoichi Enatsu), nakata@bcamath.org (Yukihiko Nakata), ymuroya@waseda.jp (Yoshiaki Muroya)
} 
Recently, Nakata et al. [16] studied the following SIRS epidemic model with a bilinear incidence rate and distributed delays,

$$
\left\{\begin{array}{l}
\frac{d S(t)}{d t}=B-\mu S(t)-\beta S(t) \int_{0}^{h} f(\tau) I(t-\tau) d \tau+\delta R(t) \\
\frac{d I(t)}{d t}=\beta S(t) \int_{0}^{h} f(\tau) I(t-\tau) d \tau-(\mu+\gamma) I(t) \\
\frac{d R(t)}{d t}=\gamma I(t)-(\mu+\delta) R(t)
\end{array}\right.
$$

$S(t), I(t)$ and $R(t)$ denote the fractions of susceptible, infective and recovered individuals at time $t$, respectively. The positive constant $B$ represents the birth rate of the population and the positive constant $\mu$ represents the death rate of susceptible, infected and recovered individuals. The positive constant $\gamma$ represents the recovery rate of infectives and the nonnegative constant $\delta$ denotes the rate at which recovered individuals lose immunity and return to susceptible class. The positive constant $\beta$ is the contact rate between susceptible and infective individuals and $h$ is a superior limit of incubation times. The incubation period distribution $f(\tau)$, which denotes the fraction of vector population in which the time taken to become infectious is $\tau$, is assumed to be continuous on $[0, h]$ satisfying $\int_{0}^{h} f(\tau) d \tau=1$ and $f(\tau) \geq 0$ for $0 \leq \tau \leq h$ (see, e.g., $\left.[1,2,17]\right)$.

By applying Lyapunov functional techniques which is an extension of those in McCluskey $[12,13]$ and the property that the total population of the system (1.1) converges to a positive constant, Nakata et al. [16] established that if $1<\tilde{R}_{0} \leq 1+\frac{\mu}{\gamma}$, then a unique endemic equilibrium of system (1.1) is globally asymptotically stable for any $\delta \geq 0$, where $\tilde{R}_{0}=\frac{\beta B}{\mu(\mu+\gamma)}$ is the basic reproduction number of system (1.1). Otherwise, they offered sufficient conditions $0 \leq \delta \leq$ $\frac{\mu}{\frac{\tilde{R}_{0}}{1+\frac{\mu}{2}}-1}$ such that the endemic equilibrium is globally asymptotically stable.

On the other hand, in modeling of those communicable diseases, nonlinear incidence rates have played a vital role in ensuring that the model can give a reasonable qualitative description for the disease dynamics such as cholera epidemic spread in Bari in 1973 (see, e.g., Capasso and Serio [3]).

Based on their idea, $\mathrm{Xu}$ and $\mathrm{Ma}$ [20] investigated the global dynamics for a delayed SIRS epidemic model with a saturated incidence rate $\frac{\beta S(t) I(t-\tau)}{1+\alpha I(t-\tau)}$ and established the global stability of the disease-free equilibrium and a sufficient condition under which the endemic equilibrium is globally asymptotically stable by applying monotone iterative techniques on a limit system obtained from the fact that the total population $N(t)=S(t)+I(t)+R(t)$ converges to a positive constant (see $\mathrm{Xu}$ and $\mathrm{Ma}$ [20, Theorem 3.1]).

In this paper, by using the key properties of Lyapunov functional techniques in Nakata et al. [16], we establish the global asymptotic stability of a disease-free equilibrium and sufficient conditions of the rate of immunity loss for the global asymptotic stability of an endemic equilibrium for the following SIRS epidemic 
models with a class of nonlinear incidence rates and distributed delays:

$$
\left\{\begin{array}{l}
\frac{d S(t)}{d t}=B-\mu_{1} S(t)-\beta S(t) \int_{0}^{h} f(\tau) G(I(t-\tau)) d \tau+\delta R(t) \\
\frac{d I(t)}{d t}=\beta S(t) \int_{0}^{h} f(\tau) G(I(t-\tau)) d \tau-\left(\mu_{2}+\gamma\right) I(t) \\
\frac{d R(t)}{d t}=\gamma I(t)-\left(\mu_{3}+\delta\right) R(t) .
\end{array}\right.
$$

and offer a unified construction of the Lyapunov functionals for both cases that the basic reproduction number is less than or equal and larger than 1 .

The initial condition of system (1.2) is given as follows.

$$
\left\{\begin{array}{l}
S(\theta)=\phi_{1}(\theta), I(\theta)=\phi_{2}(\theta), R(\theta)=\phi_{3}(\theta) \\
\phi_{i}(\theta) \geq 0, \theta \in[-h, 0], \phi_{i}(0)>0, \phi_{i} \in C\left([-h, 0], \mathbb{R}^{+}\right), i=1,2,3 .
\end{array}\right.
$$

For system (1.2), the positive constants $\mu_{1}, \mu_{2}$ and $\mu_{3}$ satisfying $\mu_{1} \leq$ $\min \left\{\mu_{2}, \mu_{3}\right\}$ represent the death rate of susceptible, infected and recovered individuals, respectively. For the incidence function $G$, we assume the following.

(H1) $G(I)$ is continuous and monotone increasing on $[0,+\infty)$ with $G(0)=0$.

(H2) $I / G(I)$ is monotone increasing on $(0,+\infty)$ with $\lim _{I \rightarrow+0}(I / G(I))=1$.

We note that $G$ is Lipschitz continuous on $[0,+\infty)$ satisfying $0<G(I) \leq I$ for all $I>0$. Under the hypotheses (H1) and (H2), system (1.2) always has a disease-free equilibrium $E^{0}=\left(S^{0}, I^{0}, R^{0}\right)$, where $S^{0}=\frac{B}{\mu_{1}}$ and $I^{0}=R^{0}=0$. In addition, if $R_{0}>1$, then system (1.2) has a unique endemic equilibrium $E^{*}=\left(S^{*}, I^{*}, R^{*}\right)$, where $S^{*}>0, I^{*}>0$ and $R^{*}>0$ (see Lemma 2.2 ).

The basic reproduction number of system (1.2) becomes as follows.

$$
R_{0}=\frac{\beta B}{\mu_{1}\left(\mu_{2}+\gamma\right)}
$$

$\frac{1}{\mu_{2}+\gamma}$ denotes the average infection period and the relation that $\lim _{I \rightarrow+0} \frac{\beta S^{0} G(I)}{I}=$ $\beta S^{0}=\beta \frac{B}{\mu_{1}}$ implies that $\beta \frac{B}{\mu_{1}}$ denotes the number of new cases infected per unit time by one infective individual at an initial infection state. Thus, $R_{0}$ denotes the expected number of secondary infectious cases generated by one typical primary case in an entirely susceptible and sufficiently large population.

If $G(I)=I$, then the incidence rate becomes a bilinear form, which is proposed in [23] for the case $\mu_{1}=\mu_{3}=\mu>0, \mu_{2}=\mu+c>0$, where $c>0$ denotes the disease-related death rate and [16] for the case $\mu_{1}=\mu_{2}=\mu_{3}=\mu>$ 0 . Moreover, if $G(I)=\frac{I}{1+\alpha I}$, then the incidence rate describes saturated effects of the prevalence of infectious diseases, which is proposed in [20] for the case $\mu_{1}=\mu_{2}=\mu_{3}=\mu>0$.

Our main results are as follows. 
Theorem 1.1 Let $R_{0}>1$. If

$$
\mu_{1} S^{*}-\delta R^{*} \geq 0,
$$

then the endemic equilibrium $E^{*}$ of system (1.2) is globally asymptotically stable. Moreover, (1.5) holds if the following conditions are satisfied.

$$
\begin{cases}0 \leq \delta<+\infty, & \text { for } 1<R_{0} \leq 1+\frac{\mu_{2}}{\gamma}, \\ 0 \leq \delta \leq \bar{\delta}:=\frac{\mu_{3}}{\frac{R_{0}}{1+\frac{\mu_{2}}{\gamma}}-1}, & \text { for } \quad R_{0}>1+\frac{\mu_{2}}{\gamma} .\end{cases}
$$

In particular, for the case $G(I)=I$, then (1.5) is equivalent to (1.6).

Theorem 1.2 If $R_{0} \leq 1$, then the disease-free equilibrium $E^{0}$ of system (1.2) is globally asymptotically stable.

To prove Theorems 1.1 and 1.2 , for $E=(S, I, R)$ and $N=S+I+R$, we define

$$
U_{\delta}^{E}(t)=\left\{\begin{aligned}
& S g\left(\frac{S(t)}{S}\right)+I g\left(\frac{I(t)}{I}\right)+\beta S G(I) \int_{0}^{h} f(\tau) \int_{t-\tau}^{t} g\left(\frac{G(I(u))}{G(I)}\right) d u d \tau \\
& \quad+\frac{\delta}{\gamma S} \frac{(R(t)-R)^{2}}{2}+\frac{\delta \gamma}{\left\{\gamma\left(\mu_{3}-\mu_{1}\right)+\left(\mu_{2}-\mu_{1}\right)\left(\mu_{1}+\mu_{3}+\delta\right)\right\} S} \frac{\left\{N(t)-N+\frac{\mu_{2}-\mu_{1}}{\gamma}(R(t)-R)\right\}^{2}}{2}, \\
& \text { if either } \mu_{1}<\mu_{2} \text { or } \mu_{1}<\mu_{3}, S g\left(\frac{S(t)}{S}\right)+I g\left(\frac{I(t)}{I}\right)+\beta S G(I) \int_{0}^{h} f(\tau) \int_{t-\tau}^{t} g\left(\frac{G(I(u))}{G(I)}\right) d u d \tau \\
& \quad+\frac{\delta}{\gamma S} \frac{(R(t)-R)^{2}}{2}+\frac{\delta}{4 \mu_{1} S} \frac{(N(t)-N)^{2}}{2}, \\
& \text { if } \quad \mu_{1}=\mu_{2}=\mu_{3},
\end{aligned}\right.
$$

where

$$
N(t)=S(t)+I(t)+R(t), \quad \text { and } \quad g(x)=x-1-\ln x \geq g(1)=0 .
$$

We offer a unified construction of Lyapunov functionals in the proof of global stability of the global stability of the disease-free equilibrium $E^{0}$ for $R_{0} \leq 1$ and the endemic equilibrium $E^{*}$ for $R_{0}>1$, respectively as follows (see Section 4);

$$
U_{\delta}^{E^{0}}(t):=\lim _{E \rightarrow E^{0}} U_{\delta}^{E}(t), \text { and } U_{\delta}^{E^{*}}(t):=\lim _{E \rightarrow E^{*}} U_{\delta}^{E}(t) .
$$

By using the relation

$$
\lim _{x \rightarrow+0} x g\left(\frac{y}{x}\right)=y, \text { for any fixed } y>0,
$$

we obtain that, for $N^{0}=S^{0}+I^{0}+R^{0}=S^{0}$ and $N^{*}=S^{*}+I^{*}+R^{*}$,

$$
U_{\delta}^{E^{0}}(t)=\left\{\begin{aligned}
& S^{0} g\left(\frac{S(t)}{S^{0}}\right)+I(t)+\beta S^{0} \int_{0}^{h} f(\tau) \int_{t-\tau}^{t} G(I(u)) d u d \tau \\
&+\frac{\delta}{\gamma S^{0}} \frac{\left(R(t)-R^{0}\right)^{2}}{2}+\frac{\delta \gamma}{\left\{\gamma\left(\mu_{3}-\mu_{1}\right)+\left(\mu_{2}-\mu_{1}\right)\left(\mu_{1}+\mu_{3}+\delta\right)\right\} S^{0}} \frac{\left\{\left(N(t)-N^{0}\right)+\frac{\mu_{2}-\mu_{1}}{\gamma}\left(R(t)-R^{0}\right)\right\}^{2}}{2}, \\
& \text { if either } \mu_{1}<\mu_{2} \quad \text { or } \mu_{1}<\mu_{3}, \\
& S^{0} g\left(\frac{S(t)}{S^{0}}\right)+I(t)+\beta S^{0} \int_{0}^{h} f(\tau) \int_{t-\tau}^{t} G(I(u)) d u d \tau \\
& \quad+\frac{\delta}{\gamma S^{0}} \frac{\left(R(t)-R^{0}\right)^{2}}{2}+\frac{\delta}{4 \mu_{1} S^{0}} \frac{\left(N(t)-N^{0}\right)^{2}}{2}, \\
& \text { if } \quad \mu_{1}=\mu_{2}=\mu_{3},
\end{aligned}\right.
$$


and

$$
U_{\delta}^{E^{*}}(t)=\left\{\begin{aligned}
& S^{*} g\left(\frac{S(t)}{S^{*}}\right)+I^{*} g\left(\frac{I(t)}{I^{*}}\right)+\beta S^{*} G\left(I^{*}\right) \int_{0}^{h} f(\tau) \int_{t-\tau}^{t} g\left(\frac{G(I(u))}{G\left(I^{*}\right)}\right) d u d \tau \\
& \quad+\frac{\delta}{\gamma S^{*}} \frac{\left(R(t)-R^{*}\right)^{2}}{2}+\frac{\delta \gamma}{\left\{\gamma\left(\mu_{3}-\mu_{1}\right)+\left(\mu_{2}-\mu_{1}\right)\left(\mu_{1}+\mu_{3}+\delta\right)\right\} S^{*}} \frac{\left\{\left(N(t)-N^{*}\right)+\frac{\mu_{2}-\mu_{1}}{\gamma}\left(R(t)-R^{*}\right)\right\}^{2}}{2}, \\
& \text { if either } \mu_{1}<\mu_{2} \text { or } \mu_{1}<\mu_{3}, \\
& S^{*} g\left(\frac{S(t)}{S^{*}}\right)+I^{*} g\left(\frac{I(t)}{I^{*}}\right)+\beta S^{*} G\left(I^{*}\right) \int_{0}^{h} f(\tau) \int_{t-\tau}^{t} g\left(\frac{G(I(u))}{G\left(I^{*}\right)}\right) d u d \tau \quad+\frac{\delta}{\gamma S^{*}} \frac{\left(R(t)-R^{*}\right)^{2}}{2}+\frac{\delta}{4 \mu_{1} S^{*}} \frac{\left(N(t)-N^{*}\right)^{2}}{2}, \\
& \text { if } \quad \mu_{1}=\mu_{2}=\mu_{3} .
\end{aligned}\right.
$$

The organization of this paper is as follows. In Section 2, some basic results are offered. In Section 3, we introduce the essential ideas of Lyapunov functional technique in McCluskey [12]. In Section 4, we establish the global asymptotic stability of the disease-free equilibrium $E^{0}$ and the endemic equilibrium $E^{*}$ of system (1.2) for $R_{0} \leq 1$ and $R_{0}>1$, respectively. Finally, we offer a conclusion in Section 5.

\section{Basic results}

In this section, we state some basic results of system (1.2). Let $\bar{\mu}=\max \left\{\mu_{2}, \mu_{3}\right\}$.

Lemma 2.1 For system (1.2) with the initial condition (1.3),

$$
\limsup _{t \rightarrow+\infty} N(t) \leq \frac{B}{\mu_{1}}, \liminf _{t \rightarrow+\infty} N(t) \geq \frac{B}{\bar{\mu}}
$$

and

$$
\left\{\begin{array}{l}
\liminf _{t \rightarrow+\infty} N(t) \geq \frac{B}{\mu_{1}}-\frac{\mu_{2}-\mu_{1}}{\mu_{1}} \limsup _{t \rightarrow+\infty} I(t)-\frac{\mu_{3}-\mu_{1}}{\mu_{1}} \limsup _{t \rightarrow+\infty} R(t), \\
\limsup _{t \rightarrow+\infty} N(t) \leq \frac{B}{\mu_{1}}-\frac{\mu_{2}-\mu_{1}}{\mu_{1}} \liminf _{t \rightarrow+\infty} I(t)-\frac{\mu_{3}-\mu_{1}}{\mu_{1}} \liminf _{t \rightarrow+\infty} R(t) .
\end{array}\right.
$$

Proof. It follows from system (1.2) that

$$
\frac{d N(t)}{d t}=B-\mu_{1} S(t)-\mu_{2} I(t)-\mu_{3} R(t) \leq B-\mu_{1} N(t),
$$

from which we obtain the first equation of (2.1). Similarly, from

$$
\frac{d N(t)}{d t} \geq B-\bar{\mu} N(t)
$$

we obtain the second equation of (2.1). Moreover, we have

$$
\frac{d N(t)}{d t}=B-\mu_{1} N(t)-\left(\mu_{2}-\mu_{1}\right) I(t)-\left(\mu_{3}-\mu_{1}\right) R(t) .
$$


First, we suppose that $N(t)$ is eventually monotone decreasing for $t \geq 0$. Then there exists a $\lim _{t \rightarrow+\infty} N(t)=N^{*}>0$ and we have

$$
0=B-\mu_{1} N^{*}-\lim _{t \rightarrow+\infty}\left\{\left(\mu_{2}-\mu_{1}\right) I(t)-\left(\mu_{3}-\mu_{1}\right) R(t)\right\}
$$

from which we obtain $(2.2)$.

Second, we suppose that $N(t)$ is not eventually monotone decreasing for $t \geq 0$. Then, there exists a sequence $\left\{t_{n}\right\}_{n=1}^{\infty}$ such that

$$
\left.\frac{d N(t)}{d t}\right|_{t=t_{n}} \geq 0, \quad \lim _{n \rightarrow+\infty} N\left(t_{n}\right)=\limsup _{t \rightarrow+\infty} N(t) .
$$

Then, from (2.4) at $t=t_{n}$, we obtain the second equation of (2.2). Similarly, we can obtain the first equation of (2.2). This completes the proof.

Lemma 2.2 (Cf. Enatsu et al. [7]) If $R_{0}>1$, then system (1.2) has a unique endemic equilibrium $E^{*}=\left(S^{*}, I^{*}, R^{*}\right)$ satisfying the following equations.

$$
\left\{\begin{array}{l}
B-\mu_{1} S^{*}-\beta S^{*} G\left(I^{*}\right)+\delta R^{*}=0 \\
\beta S^{*} G\left(I^{*}\right)-\left(\mu_{2}+\gamma\right) I^{*}=0 \\
\gamma I^{*}-\left(\mu_{3}+\delta\right) R^{*}=0
\end{array}\right.
$$

Proof. From the second and the third equations of (2.4), the following equations hold.

$$
S^{*}=\frac{\left(\mu_{2}+\gamma\right) I^{*}}{\beta G\left(I^{*}\right)}, R^{*}=\frac{\gamma I^{*}}{\mu_{3}+\delta} .
$$

After substituting (2.5) into the first equation of (2.4), we consider the following equation:

$$
H(I) \equiv B-\frac{\mu_{1}\left(\mu_{2}+\gamma\right) I}{\beta G(I)}-\left(\mu_{2}+\gamma\right) I+\frac{\gamma \delta I}{\mu_{3}+\delta}=0
$$

By the hypothesis $(\mathrm{H} 2), H(I)$ is a strictly monotone decreasing function on $(0,+\infty)$ satisfying

$$
\lim _{I \rightarrow+0} H(I)=B-\frac{\mu_{1}\left(\mu_{2}+\gamma\right)}{\beta}=B\left(1-\frac{1}{R_{0}}\right)>0,
$$

and $H(I)<0$ holds for all $I \geq B /\left\{\mu_{2}+\gamma\left(1-\frac{\delta}{\mu_{3}+\delta}\right)\right\}$. Hence, there exists a unique positive $I^{*}>0$ such that $H\left(I^{*}\right)=0$. By $(2.5)$, we obtain the conclusion of this theorem.

First, we prepare the following basic lemma.

Lemma 2.3 (Cf. Enatsu et al. [5]) Assume that $I(s) \leq I^{*}$ for any s such that $t-h \leq s<t$. If $I(t)<I(s)$ for any $s$ such that $t-h \leq s<t$ then $S(t) \leq S^{*}$. Inversely, if $S(t)>S^{*}$, then there exists an $s_{t} \in[t-h, t)$ such that $I(t) \geq I\left(s_{t}\right)$. 
Proof. Assume that $I(t)<I(s) \leq I^{*}$ holds for any $s$ such that $t-h \leq s<t$. Then, by the monotonicity of $\frac{I}{G(I)}$ in the hypothesis (H2), we have

$$
\begin{aligned}
I^{\prime}(t) & =\beta S(t) \int_{0}^{h} f(\tau) G(I(t-\tau)) d \tau-\left(\mu_{2}+\gamma\right) I(t) \\
& \geq \int_{0}^{h} f(\tau)\left\{\beta S(t) G(I(t-\tau))-\left(\mu_{2}+\gamma\right) I(t-\tau)\right\} d \tau \\
& =\int_{0}^{h} f(\tau)\left\{\beta S(t) \frac{G(I(t-\tau))}{I(t-\tau)}-\left(\mu_{2}+\gamma\right)\right\} I(t-\tau) d \tau \\
& \geq \int_{0}^{h} f(\tau)\left\{\beta S(t) \frac{G\left(I^{*}\right)}{I^{*}}-\left(\mu_{2}+\gamma\right)\right\} I(t-\tau) d \tau \\
& =\beta \frac{G\left(I^{*}\right)}{I^{*}}\left(S(t)-S^{*}\right) \int_{0}^{h} f(\tau) I(t-\tau) d \tau .
\end{aligned}
$$

Then, by $I^{\prime}(t) \leq 0$, we hence obtain $S(t) \leq S^{*}$. The remaining part of the proof is evident.

By applying Lemma 2.3, we now offer a simplified proof for the permanence of system (1.2) than that of Wang [18] (see also Xu and Ma [20]).

Lemma 2.4 If $R_{0}>1$, then for any solution of system (1.2) with initial condition (1.3), it holds that

$$
\left\{\begin{array}{l}
\liminf _{t \rightarrow+\infty} S(t) \geq v_{1}:=\frac{B}{\mu_{1}+\beta G\left(B / \mu_{1}\right)}>0, \\
\liminf _{t \rightarrow+\infty} I(t) \geq v_{2}(q):=q G\left(I^{*}\right) \mathrm{e}^{-\left(\mu_{2}+\gamma\right) \rho(q)}>0, \\
\liminf _{t \rightarrow+\infty} R(t) \geq v_{3}(q):=\frac{\gamma}{\mu_{3}+\delta} v_{2}(q)>0,
\end{array}\right.
$$

where for any $0<q<1, \rho(q)>0$ is a constant such that

$$
S^{*}<S^{\triangle}:=\frac{B}{r}\left(1-\mathrm{e}^{-r \rho(q)}\right), \quad \text { and } \quad r=\mu_{1}+\beta q G\left(I^{*}\right) .
$$

Proof. Let $(S(t), I(t), R(t))$ be any solution of system (1.2) with initial condition (1.3). By Lemma 2.1, we have $\lim \sup _{t \rightarrow+\infty} I(t) \leq B / \mu_{1}$. Hence, for $\epsilon>0$ sufficiently small, there is a $T_{1}>0$ such that $I(t)<B / \mu_{1}+\epsilon$ for $t>T_{1}$. Then, by the first equation of (1.2), we derive

$$
\frac{d S(t)}{d t} \geq B-\left\{\mu_{1}+\beta G\left(B / \mu_{1}+\epsilon\right)\right\} S(t),
$$

which implies

$$
\liminf _{t \rightarrow+\infty} S(t) \geq \frac{B}{\mu_{1}+\beta G\left(B / \mu_{1}+\epsilon\right)} .
$$


Since (2.7) holds for arbitrary $\epsilon>0$, we get $\liminf _{t \rightarrow+\infty} S(t)=v_{1}$.

We now show that $\liminf _{t \rightarrow+\infty} I(t) \geq v_{2}(q)$ for any $0<q<1$. It follows from (2.4) that $S^{*}=\frac{B}{\mu_{1}+\beta G\left(I^{*}\right)}<\frac{B}{\mu_{1}+\beta q G\left(I^{*}\right)}=\frac{B}{r}$ for any $0<q<1$. Thus, there exists a positive constant $\rho(q)$ such that (2.6) holds. We claim that it is not possible that for any solution of system (1.2), there exists a nonnegative constant $t_{0}$ such that $I(t) \leq q G\left(I^{*}\right)$ for all $t \geq t_{0}$. Suppose on the contrary that there exists a nonnegative constant $t_{0}$ such that $I(t) \leq q G\left(I^{*}\right)$ for all $t \geq t_{0}$. Then, by the hypothesis (H1), $G(I(t)) \leq q G\left(I^{*}\right)$ holds for all $t \geq t_{0}$. This yields

$$
\frac{d S(t)}{d t} \geq B-\left(\mu_{1}+\beta q G\left(I^{*}\right)\right) S(t)=B-r S(t) \quad \text { for all } t \geq t_{0}+h,
$$

which yields

$$
S(t) \geq \mathrm{e}^{-r\left(t-t_{0}\right)}\left(S\left(t_{0}\right)+B \int_{t_{0}}^{t} \mathrm{e}^{r\left(\theta-t_{0}\right)} d \theta\right) \geq \frac{B}{r}\left(1-\mathrm{e}^{-r\left(t-t_{0}\right)}\right)
$$

for any $t \geq t_{0}+h$. Therefore, we have

$$
S(t) \geq \frac{B}{r}\left(1-\mathrm{e}^{-r \rho(q)}\right)=S^{\triangle}>S^{*}
$$

for any $t \geq t_{0}+h+\rho(q)$. By the second part of Lemma 2.3, we obtain $I^{\prime}(t) \geq 0$ and for any $t \geq t_{0}+h+\rho(q)$, there exists an $s_{t} \in[t-h, t)$ such that $I(t) \geq I\left(s_{t}\right)$. For a positive constant $\hat{I}=\min _{t_{0}+\rho(q) \leq s \leq t_{0}+h+\rho(q)} I(s)$, we then have

$$
I(t) \geq \hat{I} \quad \text { for any } t \geq t_{0}+h+\rho(q) .
$$

We here consider the following functional.

$$
W(t)=I(t)+\beta \int_{0}^{h} f(\tau) \int_{t-\tau}^{t} S(u+\tau) G(I(u)) d u d \tau .
$$

For $t \geq t_{0}+h+\rho(q)$, we have

$$
\begin{aligned}
\frac{d W(t)}{d t}= & \beta S(t) \int_{0}^{h} f(\tau) G(I(t-\tau)) d \tau-\left(\mu_{2}+\gamma\right) I(t) \\
& +\beta \int_{0}^{h} f(\tau)\{S(t+\tau) G(I(t))-S(t) G(I(t-\tau))\} d \tau \\
= & \beta \int_{0}^{h} f(\tau) S(t+\tau) G(I(t)) d \tau-\left(\mu_{2}+\gamma\right) I(t) \\
= & \left\{\beta S^{\triangle} \frac{G(I(t))}{I(t)}-\left(\mu_{2}+\gamma\right)\right\} I(t) \\
> & \left\{\beta S^{\triangle} \frac{G\left(I^{*}\right)}{I^{*}}-\left(\mu_{2}+\gamma\right)\right\} I(t) \\
> & \beta\left(S^{\triangle}-S^{*}\right) \frac{G\left(I^{*}\right)}{I^{*}} \hat{I}>0
\end{aligned}
$$


which implies $\lim _{t \rightarrow+\infty} W(t)=+\infty$. However, by Lemma 2.1, it holds that $\lim \sup _{t \rightarrow+\infty} W(t) \leq \frac{B}{\mu_{1}}+\beta \frac{B}{\mu_{1}} G\left(\frac{B}{\mu_{1}}\right)<+\infty$. This is a contradiction. Hence, the claim is proved.

By the claim, we are left to consider two cases. First, $I(t) \geq q G\left(I^{*}\right)$ for all $t$ sufficiently large. Second, $I(t)$ oscillates about $q G\left(I^{*}\right)$ for all $t$ sufficiently large. If the first case holds, then we get the conclusion of the proof. If the second case holds, then we can choose $t_{1}$ and $t_{2}\left(t_{1}<t_{2}\right)$ sufficiently large such that

$$
I\left(t_{1}\right)=I\left(t_{2}\right)=q G\left(I^{*}\right), \quad \text { and } \quad I(t)<q G\left(I^{*}\right)
$$

for $t_{1}<t<t_{2}$. Since $\frac{d I(t)}{d t} \geq-\left(\mu_{2}+\gamma\right) I(t)$ for $t \geq t_{1}$, we have

$$
I(t) \geq I\left(t_{1}\right) \mathrm{e}^{-\left(\mu_{2}+\gamma\right)\left(t-t_{1}\right)} \geq q G\left(I^{*}\right) \mathrm{e}^{-\left(\mu_{2}+\gamma\right)\left(t-t_{1}\right)}
$$

for any $t \geq t_{1}$. Therefore, we obtain

$$
I(t) \geq q G\left(I^{*}\right) \mathrm{e}^{-\left(\mu_{2}+\gamma\right) \rho(q)}=v_{2}(q)
$$

for $t_{1} \leq t \leq t_{1}+\rho(q)$. If $t_{2} \geq t_{1}+\rho(q)$, then by applying the similar discussion to (2.8) and (2.9) in place of $t_{0}$ by $t_{1}$, we obtain $I(t) \geq v_{2}(q)$ for $t_{1}+\rho(q) \leq t \leq t_{2}$. Hence, we prove $I(t) \geq v_{2}(q)$ for $t_{1} \leq t \leq t_{2}$. Since the interval $t_{1} \leq t \leq t_{2}$ is arbitrarily chosen, we conclude that $I(t) \geq v_{2}(q)$ for all sufficiently large. Since $q$ is also arbitrarily chosen, Thus, we obtain $\liminf _{t \rightarrow+\infty} I(t) \geq v_{2}(q)$, which implies $\liminf \operatorname{in}_{t \rightarrow+\infty} R(t) \geq v_{3}(q)$. This completes the proof.

By Lemmas 2.1 and 2.4, we obtain the permanence of system (1.2) for $R_{0}>1$.

\section{Lyapunov functional techniques in McCluskey [12]}

In this section, we consider the case $\delta=0$ for system (1.2). Then system (1.2) becomes an SIR epidemic model with a class of nonlinear incidence rates and distributed delays as follows.

$$
\left\{\begin{array}{l}
\frac{d S(t)}{d t}=B-\mu_{1} S(t)-\beta S(t) \int_{0}^{h} f(\tau) G(I(t-\tau)) d \tau \\
\frac{d I(t)}{d t}=\beta S(t) \int_{0}^{h} f(\tau) G(I(t-\tau)) d \tau-\left(\mu_{2}+\gamma\right) I(t), \\
\frac{d R(t)}{d t}=\gamma I(t)-\mu_{3} R(t) .
\end{array}\right.
$$

We consider the following Lyapunov functionals.

$$
\left\{\begin{array}{l}
U_{0}^{E^{0}}(t)=S^{0} g\left(\frac{S(t)}{S^{0}}\right)+I(t)+\beta S^{0} \int_{0}^{h} f(\tau) \int_{t-\tau}^{t} G(I(u)) d u d \tau \\
U_{0}^{E^{*}}(t)=S^{*} g\left(\frac{S(t)}{S^{*}}\right)+I^{*} g\left(\frac{I(t)}{I^{*}}\right)+\beta S^{*} G\left(I^{*}\right) \int_{0}^{h} f(\tau) \int_{t-\tau}^{t} g\left(\frac{G(I(u))}{G\left(I^{*}\right)}\right) d u d \tau .
\end{array}\right.
$$

We introduce essential ideas of the global stability of the endemic equilibrium $E^{*}$ of (3.1) for $R_{0}>1$ in McCluskey [12]. For a fixed $0 \leq \tau \leq h$, we put

$$
x_{t}=\frac{S(t)}{S^{*}}, y_{t}=\frac{I(t)}{I^{*}}, \tilde{y}_{t}=\frac{G(I(t))}{G\left(I^{*}\right)}, \tilde{y}_{t, \tau}=\frac{G(I(t-\tau))}{G\left(I^{*}\right)} .
$$


Then, we obtain

$$
\begin{aligned}
\frac{d}{d t}\left\{g\left(\frac{S(t)}{S^{*}}\right)\right\} & =\left(\frac{1}{S^{*}}-\frac{1}{S(t)}\right)\left\{B-\mu_{1} S(t)-\beta S(t) \int_{0}^{h} f(\tau) G(I(t-\tau)) d \tau\right\} \\
& =\frac{S(t)-S^{*}}{S^{*} S(t)}\left\{B-\mu_{1} S(t)-\beta S(t) \int_{0}^{h} f(\tau) G(I(t-\tau)) d \tau\right\}(3.3)
\end{aligned}
$$

Substituting $B=\mu_{1} S^{*}+\beta S^{*} G\left(I^{*}\right)$ in $(3.3)$,

$$
\begin{aligned}
& \frac{d}{d t}\left\{g\left(\frac{S(t)}{S^{*}}\right)\right\} \\
= & \frac{S(t)-S^{*}}{S^{*} S(t)}\left\{\left(\mu_{1} S^{*}+\beta S^{*} G\left(I^{*}\right)\right)-\mu_{1} S(t)-\beta S(t) \int_{0}^{h} f(\tau) G(I(t-\tau)) d \tau\right\} \\
= & \frac{S(t)-S^{*}}{S^{*} S(t)}\left\{-\mu_{1}\left(S(t)-S^{*}\right)+\beta \int_{0}^{h} f(\tau)\left(S^{*} G\left(I^{*}\right)-S(t) G(I(t-\tau))\right) d \tau\right\} \\
= & -\mu_{1}\left(1-\frac{S^{*}}{S(t)}\right)\left(\frac{S(t)}{S^{*}}-1\right) \\
& +\beta G\left(I^{*}\right) \int_{0}^{h} f(\tau)\left(1-\frac{S^{*}}{S(t)}\right)\left(1-\frac{S(t)}{S^{*}} \frac{G(I(t-\tau))}{G\left(I^{*}\right)}\right) d \tau \\
= & -\mu_{1}\left(1-\frac{1}{x_{t}}\right)\left(x_{t}-1\right)+\beta G\left(I^{*}\right) \int_{0}^{h} f(\tau)\left(1-\frac{1}{x_{t}}\right)\left(1-x_{t} \tilde{y}_{t, \tau}\right) d \tau . \quad(3.4)
\end{aligned}
$$

Similar to the above discussion, by the relation $\mu_{2}+\gamma=\frac{\beta S^{*} G\left(I^{*}\right)}{I^{*}}$, we have

$$
\begin{aligned}
\frac{d}{d t}\left\{g\left(\frac{I(t)}{I^{*}}\right)\right\} & =\frac{I(t)-I^{*}}{I^{*} I(t)}\left\{\beta S(t) \int_{0}^{h} f(\tau) G(I(t-\tau)) d \tau-\left(\mu_{2}+\gamma\right) I(t)\right\} \\
& =\frac{I(t)-I^{*}}{I^{*} I(t)}\left\{\beta S(t) \int_{0}^{h} f(\tau) G(I(t-\tau)) d \tau-\beta S^{*} \frac{G\left(I^{*}\right)}{I^{*}} I(t)\right\} \\
& =\beta S^{*} \frac{G\left(I^{*}\right)}{I^{*}} \int_{0}^{h} f(\tau)\left(1-\frac{I^{*}}{I(t)}\right)\left(\frac{S(t)}{S^{*}} \frac{G(I(t-\tau))}{G\left(I^{*}\right)}-\frac{I(t)}{I^{*}}\right) d \tau \\
& =\beta S^{*} \frac{G\left(I^{*}\right)}{I^{*}} \int_{0}^{h} f(\tau)\left(1-\frac{1}{y_{t}}\right)\left(x_{t} \tilde{y}_{t, \tau}-y_{t}\right) d \tau
\end{aligned}
$$

Finally, we obtain

$$
\frac{d}{d t}\left\{\int_{0}^{h} f(\tau) \int_{t-\tau}^{t} g\left(\frac{G(I(u))}{G\left(I^{*}\right)}\right) d u d \tau\right\}=\int_{0}^{h} f(\tau)\left(g\left(\tilde{y}_{t}\right)-g\left(\tilde{y}_{t, \tau}\right)\right) d \tau .
$$

The following lemma plays an important role to apply techniques of equation deformation in McCluskey [12] to the global stability analysis for the endemic equilibria of system (1.2). 
Lemma 3.1 If $R_{0}>1$, then it holds that

$$
\begin{aligned}
& \left(1-\frac{1}{x_{t}}\right)\left(1-x_{t} \tilde{y}_{t, \tau}\right)+\left(1-\frac{1}{y_{t}}\right)\left(x_{t} \tilde{y}_{t, \tau}-y_{t}\right) \\
= & -g\left(\frac{1}{x_{t}}\right)-g\left(\frac{x_{t} \tilde{y}_{t, \tau}}{y_{t}}\right)-\left(g\left(y_{t}\right)-g\left(\tilde{y}_{t, \tau}\right)\right) .
\end{aligned}
$$

Proof.

$$
\begin{aligned}
& \left(1-\frac{1}{x_{t}}\right)\left(1-x_{t} \tilde{y}_{t, \tau}\right)+\left(1-\frac{1}{y_{t}}\right)\left(x_{t} \tilde{y}_{t, \tau}-y_{t}\right) \\
= & \left(1-\frac{1}{x_{t}}-x_{t} \tilde{y}_{t, \tau}+\tilde{y}_{t}\right)+\left(x_{t} \tilde{y}_{t, \tau}-\frac{x_{t} \tilde{y}_{t, \tau}}{y_{t}}-y_{t}+1\right) \\
= & 2-\frac{1}{x_{t}}+\tilde{y}_{t, \tau}-\frac{x_{t} \tilde{y}_{t, \tau}}{y_{t}}-y_{t} \\
= & -g\left(\frac{1}{x_{t}}\right)-g\left(\frac{x_{t} \tilde{y}_{t, \tau}}{y_{t}}\right)-\left(g\left(y_{t}\right)-g\left(\tilde{y}_{t, \tau}\right)\right) .
\end{aligned}
$$

This completes the proof.

By Lemma 3.1, the time derivative of $U_{0}^{E^{*}}(t)$ along the solution of system (3.1) becomes as follows.

$$
\begin{aligned}
\frac{d U_{0}^{E^{*}}(t)}{d t}= & -\mu_{1} S^{*} \frac{\left(x_{t}-1\right)^{2}}{x_{t}} \\
& -\beta S^{*} G\left(I^{*}\right) \int_{0}^{h} f(\tau)\left\{g\left(\frac{1}{x_{t}}\right)+g\left(\frac{x_{t} \tilde{y}_{t, \tau}}{y_{t}}\right)+\left(g\left(y_{t}\right)-g\left(\tilde{y}_{t}\right)\right)\right\} d \tau .
\end{aligned}
$$

In order to show $\frac{d U_{0}^{E^{*}}(t)}{d t} \leq 0$, we need the following lemma.

Lemma 3.2 If $R_{0}>1$, then for all $t \geq 0$,

$$
g\left(y_{t}\right)-g\left(\tilde{y}_{t}\right) \geq \frac{G(I(t))-G\left(I^{*}\right)}{I^{*}}\left(\frac{I(t)}{G(I(t))}-\frac{I^{*}}{G\left(I^{*}\right)}\right) \geq 0 .
$$

Proof. First, we have $\tilde{y}_{t}-1=\frac{G(I(t))-G\left(I^{*}\right)}{G\left(I^{*}\right)}$ and

$$
y_{t}-\tilde{y}_{t}=\frac{I(t)}{I^{*}}-\frac{G(I(t))}{G\left(I^{*}\right)}=\frac{G(I(t))}{I^{*}}\left(\frac{I(t)}{G(I(t))}-\frac{I^{*}}{G\left(I^{*}\right)}\right) .
$$

Since $g^{\prime}(x)=1-\frac{1}{x}=\frac{x-1}{x}$ and $g^{\prime \prime}(x)=\frac{1}{x^{2}}>0$ for all $x>0$, by the hypotheses (H1) and (H2), we obtain

$$
g\left(y_{t}\right)-g\left(\tilde{y}_{t}\right) \geq \frac{\tilde{y}_{t}-1}{\tilde{y}_{t}}\left(y_{t}-\tilde{y}_{t}\right)=\frac{G(I(t))-G\left(I^{*}\right)}{I^{*}}\left(\frac{I(t)}{G(I(t))}-\frac{I^{*}}{G\left(I^{*}\right)}\right) \geq 0 .
$$

Thus, we get the conclusion of this lemma. 
By Lemma 3.2, we obtain $\frac{d U_{0}^{E^{*}}(t)}{d t} \leq 0$. From the permanence result in Lemmas 2.1 and 2.4, by applying LaSalle invariance principle [11, Corollary 5.2], the endemic equilibrium $E^{*}$ of system (3.1) is globally asymptotically stable.

Similar to the case $R_{0}>1$, for $R_{0} \leq 1$, we obtain

$$
\frac{d}{d t}\left\{g\left(\frac{S(t)}{S^{0}}\right)\right\}=\frac{S(t)-S^{0}}{S^{0} S(t)}\left\{-\mu_{1}\left(S(t)-S^{0}\right)-\beta S(t) \int_{0}^{h} f(\tau) G(I(t-\tau)) d \tau\right\} .
$$

Then, we have

$$
\begin{aligned}
\frac{d U_{0}^{E^{0}}(t)}{d t}= & -\mu_{1} \frac{\left(S(t)-S^{0}\right)^{2}}{S(t)}-\beta\left(S(t)-S^{0}\right) \int_{0}^{h} f(\tau) G(I(t-\tau)) d \tau \\
& +\beta S(t) \int_{0}^{h} f(\tau) G(I(t-\tau)) d \tau-\left(\mu_{2}+\gamma\right) I(t) \\
& +\beta S^{0} \int_{0}^{h} f(\tau)\{G(I(t))-G(I(t-\tau))\} d \tau \\
= & -\mu_{1} \frac{\left(S(t)-S^{0}\right)^{2}}{S(t)}+\left\{\beta S^{0} G(I(t))-\left(\mu_{2}+\gamma\right) I(t)\right\} \\
= & -\mu_{1} \frac{\left(S(t)-S^{0}\right)^{2}}{S(t)}+\left(\mu_{2}+\gamma\right)\left(R_{0} \frac{G(I(t))}{I(t)}-1\right) I(t) \\
\leq & -\mu_{1} \frac{\left(S(t)-S^{0}\right)^{2}}{S(t)}+\left(\mu_{2}+\gamma\right)\left(R_{0}-1\right) I(t) \leq 0 .
\end{aligned}
$$

By applying Lyapunov-LaSalle asymptotic stability theorem [11, Theorem 5.3], the disease-free equilibrium $E^{0}$ of system (3.1) is globally asymptotically stable. Summarizing the above discussion, we obtain the following result.

Corollary 3.1 (See McCluskey $[12,13])$ The following statement holds true.

(I) If $R_{0} \leq 1$, then the disease-free equilibrium $E^{0}$ of system (3.1) is globally asymptotically stable.

(II) If $R_{0}>1$, then the endemic equilibrium $E^{*}$ of system (3.1) is globally asymptotically stable.

The results in Corollary 3.1 plays an important role to extend the global stability results for the case $\delta=0$ to those for the case $\delta \geq 0$. Recently, the similar global stability results for delayed SIR epidemic models with a wider class of nonlinear incidence rates are obtained in $[6,8,9,14]$. We note that the differentiability of the incidence function as imposed in $[8,9,14]$ is no longer needed. In addition, the inequality estimation in Lemma 3.2 is also extended in the Lyapunov functional techniques for a delayed SIRS model with a nonseparable incidence rate in Enatsu et al. [7].

\section{Proofs of Theorems 1.1 and 1.2}

In this section, by applying Lyapunov functional techniques for the SIR epidemic model (3.1) in Section 3, we prove Theorems 1.1 and 1.2. 
First, we consider the case $R_{0}>1$ and prove Theorem 1.1. In addition to the notations in (3.3), we put

$$
z_{t}=\frac{R(t)}{R^{*}}, n_{t}=\frac{N(t)}{N^{*}} .
$$

The following lemma also plays an important role as in Nakata et al. [16].

Lemma 4.1 Let $R_{0}>1$. Then it holds that

$$
\begin{aligned}
\left(1-\frac{1}{x_{t}}\right)\left(z_{t}-1\right)-\left(z_{t}-1\right)\left(x_{t}-1\right) & =\left(1-\frac{1}{x_{t}}\right)\left(1-x_{t}\right)\left(z_{t}-1\right) \\
& =-\frac{\left(x_{t}-1\right)^{2}}{x_{t}}\left(z_{t}-1\right) .
\end{aligned}
$$

Lemma 4.2 Let $R_{0}>1$. Then (1.5) holds if (1.6) holds. In particular, for the case $G(I)=I$, then (1.5) is equivalent to (1.6).

Proof. From (2.4), $I^{*}$ satisfies the following equation.

$$
\frac{\beta\left\{\mu_{3}\left(\mu_{2}+\gamma\right)+\mu_{2} \delta\right\}}{\mu_{3}+\delta} I^{*}+\mu_{1}\left(\mu_{2}+\gamma\right) \frac{I^{*}}{G\left(I^{*}\right)}=\beta B .
$$

From the hypothesis (H2), we have

$$
\begin{aligned}
I^{*} & =\frac{\mu_{3}+\delta}{\beta\left\{\mu_{3}\left(\mu_{2}+\gamma\right)+\mu_{2} \delta\right\}}\left\{\beta B-\mu_{1}\left(\mu_{2}+\gamma\right) \frac{I^{*}}{G\left(I^{*}\right)}\right\} \\
& \leq \frac{\mu_{3}+\delta}{\beta\left\{\mu_{3}\left(\mu_{2}+\gamma\right)+\mu_{2} \delta\right\}}\left\{\beta B-\mu_{1}\left(\mu_{2}+\gamma\right)\right\} \\
& =\frac{\mu_{1}\left(\mu_{2}+\gamma\right)\left(\mu_{3}+\delta\right)}{\beta\left\{\mu_{3}\left(\mu_{2}+\gamma\right)+\mu_{2} \delta\right\}}\left(R_{0}-1\right) .
\end{aligned}
$$

Therefore, from (1.6) and (4.3), we obtain

$$
\begin{aligned}
\mu_{1} S^{*}-\delta R^{*} & =\mu_{1} \frac{\left(\mu_{2}+\gamma\right)\left(\mu_{3}+\delta\right) R^{*}}{\beta \gamma G\left(I^{*}\right)}\left(\mu_{2}+\gamma\right)\left(\mu_{3}+\delta\right)-\delta R^{*} \\
& =\frac{R^{*}}{\beta \gamma G\left(I^{*}\right)}\left\{\mu_{1}\left(\mu_{2}+\gamma\right)\left(\mu_{3}+\delta\right)-\beta \gamma \delta G\left(I^{*}\right)\right\} \\
& \geq \frac{R^{*}}{\beta \gamma G\left(I^{*}\right)}\left\{\mu_{1}\left(\mu_{2}+\gamma\right)\left(\mu_{3}+\delta\right)-\beta \gamma \delta I^{*}\right\} \\
& \geq \frac{R^{*}}{\beta \gamma G\left(I^{*}\right)}\left\{\mu_{1}\left(\mu_{2}+\gamma\right)\left(\mu_{3}+\delta\right)-\gamma \delta \frac{\mu_{1}\left(\mu_{2}+\gamma\right)\left(\mu_{3}+\delta\right)}{\mu_{3}\left(\mu_{2}+\gamma\right)+\mu_{2} \delta}\left(R_{0}-1\right)\right\} \\
& =\frac{R^{*}}{\beta \gamma G\left(I^{*}\right)}\left[\frac{\mu_{1}\left(\mu_{2}+\gamma\right)^{2}\left(\mu_{3}+\delta\right)}{\mu_{3}\left(\mu_{2}+\gamma\right)+\mu_{2} \delta}\left\{\mu_{3}-\delta\left(\frac{R_{0}}{1+\frac{\mu_{2}}{\gamma}}-1\right)\right\}\right] \geq 0 .
\end{aligned}
$$

From the above discussion, it is obvious that (1.5) is equivalent to (1.6) for $G(I)=I$. This completes the proof. 
Proof of Theorem 1.1. We consider the following Lyapunov functional.

$U_{\delta}^{E^{*}}(t)=\left\{\begin{array}{l}U_{0}^{E^{*}}(t)+\frac{\delta}{\gamma S^{*}} \frac{\left(R(t)-R^{*}\right)^{2}}{2}+\frac{\delta \gamma}{\left\{\gamma\left(\mu_{3}-\mu_{1}\right)+\left(\mu_{2}-\mu_{1}\right)\left(\mu_{1}+\mu_{3}+\delta\right)\right\} S^{*}} \frac{\left\{\left(N(t)-N^{*}\right)+\frac{\mu_{2}-\mu_{1}}{\gamma}\left(R(t)-R^{*}\right)\right\}^{2}}{2}, \\ \text { if either } \mu_{1}<\mu_{2} \text { or } \mu_{1}<\mu_{3}, \\ U_{0}^{E^{*}}(t)+\frac{\delta}{\gamma S^{*}} \frac{\left(R(t)-R^{*}\right)^{2}}{2}+\frac{\delta}{4 \mu_{1} S^{*}} \frac{\left(N(t)-N^{*}\right)^{2}}{2}, \\ \text { if } \mu_{1}=\mu_{2}=\mu_{3},\end{array}\right.$

where $U_{0}^{E^{*}}(t)$ is defined in (3.2). First, by Lemma 3.1, the time derivative of $U_{0}^{E^{*}}(t)$ along the solution of system (1.2) becomes as follows.

$$
\begin{aligned}
\frac{d U_{0}^{E^{*}}(t)}{d t}= & -\mu_{1} S^{*} \frac{\left(x_{t}-1\right)^{2}}{x_{t}}+\delta R^{*}\left(1-\frac{1}{x_{t}}\right)\left(z_{t}-1\right) \\
& -\int_{0}^{h} f(\tau)\left\{g\left(\frac{1}{x_{t}}\right)+g\left(\frac{x_{t} \tilde{y}_{t, \tau}}{y_{t}}\right)+g\left(y_{t}\right)-g\left(\tilde{y}_{t}\right)\right\} d \tau .(4.4)
\end{aligned}
$$

Second, by $I(t)=N(t)-S(t)-R(t)$, calculating the time derivatives of $\frac{\delta}{\gamma S^{*}} \frac{\left(R(t)-R^{*}\right)^{2}}{2}$ gives

$$
\begin{aligned}
& \frac{d}{d t}\left\{\frac{\delta}{\gamma S^{*}} \frac{\left(R(t)-R^{*}\right)^{2}}{2}\right\} \\
= & \frac{\delta}{\gamma S^{*}}\left(R(t)-R^{*}\right)\left\{\gamma I(t)-\left(\mu_{3}+\delta\right) R(t)\right\} \\
= & \frac{\delta}{\gamma S^{*}}\left(R(t)-R^{*}\right)\left\{\gamma(N(t)-S(t)-R(t))-\left(\mu_{3}+\delta\right) R(t)\right\} \\
= & \frac{\delta}{\gamma S^{*}}\left(R(t)-R^{*}\right)\left\{\gamma\left(N(t)-N^{*}\right)-\gamma\left(S(t)-S^{*}\right)-\left(\mu_{3}+\gamma+\delta\right)\left(R(t)-R^{*}\right)\right\} \\
= & \frac{\delta R^{*} N^{*}}{S^{*}}\left(z_{t}-1\right)\left(n_{t}-1\right)-\delta R^{*}\left(z_{t}-1\right)\left(x_{t}-1\right)-\frac{\delta\left(\mu_{3}+\gamma+\delta\right)\left(R^{*}\right)^{2}}{\gamma S^{*}}\left(z_{t}-1\right)^{2} .
\end{aligned}
$$


For the first case either $\mu_{1}<\mu_{2}$ or $\mu_{1}<\mu_{3}$, by $S(t)=N(t)-I(t)-R(t)$, we obtain

$$
\begin{aligned}
& \frac{d}{d t}\left\{\frac{\left\{\left(N(t)-N^{*}\right)+\frac{\mu_{2}-\mu_{1}}{\gamma}\left(R(t)-R^{*}\right)\right\}^{2}}{2}\right\} \\
= & \left\{\left(N(t)-N^{*}\right)+\frac{\mu_{2}-\mu_{1}}{\gamma}\left(R(t)-R^{*}\right)\right\}\left\{B-\mu_{1} S(t)-\mu_{2} I(t)-\mu_{3} R(t)-\frac{\mu_{2}-\mu_{1}}{\gamma}\left(\gamma I(t)-\mu_{3} R(t)\right)\right\} \\
= & \left\{\left(N(t)-N^{*}\right)+\frac{\mu_{2}-\mu_{1}}{\gamma}\left(R(t)-R^{*}\right)\right\} \\
& \times\left\{B-\mu_{1}(N(t)-I(t)-R(t))-\mu_{2} I(t)-\mu_{3} R(t)-\frac{\mu_{2}-\mu_{1}}{\gamma}\left(\gamma I(t)-\mu_{3} R(t)\right)\right\} \\
= & \left\{\left(N(t)-N^{*}\right)+\frac{\mu_{2}-\mu_{1}}{\gamma}\left(R(t)-R^{*}\right)\right\} \\
& \times\left\{B-\mu_{1} N(t)-\left(\mu_{2}-\mu_{1}\right) I(t)-\left(\mu_{3}-\mu_{1}\right) R(t)-\frac{\mu_{2}-\mu_{1}}{\gamma}\left(\gamma I(t)-\left(\mu_{3}+\delta\right) R(t)\right)\right\} \\
= & \left\{\left(N(t)-N^{*}\right)+\frac{\mu_{2}-\mu_{1}}{\gamma}\left(R(t)-R^{*}\right)\right\}\left[B-\mu_{1} N(t)-\left\{\left(\mu_{3}-\mu_{1}\right)+\frac{\left(\mu_{2}-\mu_{1}\right)\left(\mu_{3}+\delta\right)}{\gamma} R(t)\right\}\right] \\
= & \left\{\left(N(t)-N^{*}\right)+\frac{\mu_{2}-\mu_{1}}{\gamma}\left(R(t)-R^{*}\right)\right\} \\
& \times\left[-\mu_{1}\left(N(t)-N^{*}\right)-\left\{\left(\mu_{3}-\mu_{1}\right)+\frac{\left(\mu_{2}-\mu_{1}\right)\left(\mu_{3}+\delta\right)}{\gamma}\right\}\left(R(t)-R^{*}\right)\right] . \\
= & -\mu_{1}\left(N^{*}\right)^{2}\left(n_{t}-1\right)^{2}-\left\{\left(\mu_{3}-\mu_{1}\right)+\frac{\left(\mu_{2}-\mu_{1}\right)\left(\mu_{1}+\mu_{3}+\delta\right)}{\gamma}\right\} N^{*} R^{*}\left(n_{t}-1\right)\left(z_{t}-1\right) \\
& -\frac{\mu_{2}-\mu_{1}}{\gamma}\left\{\left(\mu_{3}-\mu_{1}\right)+\frac{\left(\mu_{2}-\mu_{1}\right)\left(\mu_{3}+\delta\right)}{\gamma}\right\}\left(R^{*}\right)^{2}\left(z_{t}-1\right)^{2} .
\end{aligned}
$$

Combining (4.4), (4.5) and (4.6), we have

$$
\begin{aligned}
\frac{d U_{\delta}^{E^{*}}(t)}{d t}= & -\mu_{1} S^{*} \frac{\left(x_{t}-1\right)^{2}}{x_{t}}+\delta R^{*}\left(1-\frac{1}{x_{t}}\right)\left(z_{t}-1\right) \\
& +\frac{\delta R^{*} N^{*}}{S^{*}}\left(z_{t}-1\right)\left(n_{t}-1\right)-\delta R^{*}\left(z_{t}-1\right)\left(x_{t}-1\right)-\frac{\delta\left(\mu_{3}+\gamma+\delta\right)\left(R^{*}\right)^{2}}{\gamma S^{*}}\left(z_{t}-1\right)^{2} \\
& -\frac{\mu_{1} \delta \gamma\left(N^{*}\right)^{2}}{\left\{\gamma\left(\mu_{3}-\mu_{1}\right)+\left(\mu_{2}-\mu_{1}\right)\left(\mu_{1}+\mu_{3}+\delta\right)\right\} S^{*}}\left(n_{t}-1\right)^{2}-\frac{\delta N^{*} R^{*}}{S^{*}}\left(n_{t}-1\right)\left(z_{t}-1\right) \\
& -\frac{\delta\left(\mu_{2}-\mu_{1}\right)\left\{\gamma\left(\mu_{3}-\mu_{1}\right)+\left(\mu_{2}-\mu_{1}\right)\left(\mu_{3}+\delta\right)\right\}\left(R^{*}\right)^{2}}{\gamma\left\{\gamma\left(\mu_{3}-\mu_{1}\right)+\left(\mu_{2}-\mu_{1}\right)\left(\mu_{1}+\mu_{3}+\delta\right)\right\} S^{*}}\left(z_{t}-1\right)^{2} \\
& -\int_{0}^{h} f(\tau)\left\{g\left(\frac{1}{x_{t}}\right)+g\left(\frac{x_{t} \tilde{y}_{t, \tau}}{y_{t}}\right)+g\left(y_{t}\right)-g\left(\tilde{y}_{t}\right)\right\} d \tau .
\end{aligned}
$$


By the condition (1.5) and Lemma 4.1, we have

$$
\begin{aligned}
\frac{d U_{\delta}^{E^{*}}(t)}{d t}= & -\left(\mu_{1} S^{*}+\delta\left(R(t)-R^{*}\right)\right) \frac{\left(x_{t}-1\right)^{2}}{x_{t}} \\
& -\frac{\mu_{1} \delta \gamma\left(N^{*}\right)^{2}}{\left\{\gamma\left(\mu_{3}-\mu_{1}\right)+\left(\mu_{2}-\mu_{1}\right)\left(\mu_{1}+\mu_{3}+\delta\right)\right\} S^{*}}\left(n_{t}-1\right)^{2} \\
& -\left\{\frac{\delta\left(\mu_{2}-\mu_{1}\right)\left\{\gamma\left(\mu_{3}-\mu_{1}\right)+\left(\mu_{2}-\mu_{1}\right)\left(\mu_{3}+\delta\right)\right\}\left(R^{*}\right)^{2}}{\gamma\left\{\gamma\left(\mu_{3}-\mu_{1}\right)+\left(\mu_{2}-\mu_{1}\right)\left(\mu_{1}+\mu_{3}+\delta\right)\right\} S^{*}}+\frac{\delta\left(\mu_{3}+\gamma+\delta\right)\left(R^{*}\right)^{2}}{\gamma S^{*}}\right\}\left(z_{t}-1\right)^{2} \\
& -\int_{0}^{h} f(\tau)\left\{g\left(\frac{1}{x_{t}}\right)+g\left(\frac{x_{t} \tilde{y}_{t, \tau}}{y_{t}}\right)+g\left(y_{t}\right)-g\left(\tilde{y}_{t}\right)\right\} d \tau \\
\leq & -\frac{\mu_{1} \delta \gamma\left(N^{*}\right)^{2}}{\left\{\gamma\left(\mu_{3}-\mu_{1}\right)+\left(\mu_{2}-\mu_{1}\right)\left(\mu_{1}+\mu_{3}+\delta\right)\right\} S^{*}}\left(n_{t}-1\right)^{2} \\
& -\left\{\frac{\delta\left(\mu_{2}-\mu_{1}\right)\left\{\gamma\left(\mu_{3}-\mu_{1}\right)+\left(\mu_{2}-\mu_{1}\right)\left(\mu_{3}+\delta\right)\right\}\left(R^{*}\right)^{2}}{\gamma\left\{\gamma\left(\mu_{3}-\mu_{1}\right)+\left(\mu_{2}-\mu_{1}\right)\left(\mu_{1}+\mu_{3}+\delta\right)\right\} S^{*}}+\frac{\delta\left(\mu_{3}+\gamma+\delta\right)\left(R^{*}\right)^{2}}{\gamma S^{*}}\right\}\left(z_{t}-1\right)^{2} \\
& -\int_{0}^{h} f(\tau)\left\{g\left(\frac{1}{x_{t}}\right)+g\left(\frac{x_{t} \tilde{y}_{t, \tau}}{y_{t}}\right)\right\} d \tau .
\end{aligned}
$$

For the second case $\mu_{1}=\mu_{2}=\mu_{3}$, by $S^{*}+I^{*}+R^{*}=B / \mu_{1}$, we obtain

$\frac{d}{d t}\left\{\frac{\delta}{4 \mu_{1} S^{*}} \frac{\left(N(t)-N^{*}\right)^{2}}{2}\right\}=\frac{\delta}{4 \mu_{1} S^{*}}\left(N(t)-N^{*}\right)\left(B-\mu_{1} N(t)\right)=-\frac{\delta}{4 S^{*}}\left(N(t)-N^{*}\right)^{2}$.

Combining (4.4), (4.5) and (4.9), we have

$$
\begin{aligned}
\frac{d U_{\delta}^{E^{*}}(t)}{d t}= & -\mu_{1} S^{*} \frac{\left(x_{t}-1\right)^{2}}{x_{t}}+\delta R^{*}\left(1-\frac{1}{x_{t}}\right)\left(z_{t}-1\right)-\delta R^{*}\left(z_{t}-1\right)\left(x_{t}-1\right) \\
& -\frac{\delta\left(R^{*}\right)^{2}}{S^{*}}\left(z_{t}-1\right)^{2}+\frac{\delta R^{*} N^{*}}{S^{*}}\left(z_{t}-1\right)\left(n_{t}-1\right)-\frac{\delta\left(N^{*}\right)^{2}}{4 S^{*}}\left(n_{t}-1\right)^{2} \\
& -\beta S^{*} G\left(I^{*}\right) \int_{0}^{h} f(\tau)\left\{g\left(\frac{1}{x_{t}}\right)+g\left(\frac{x_{t} y_{t, \tau}}{y_{t}}\right)+g\left(y_{t}\right)-g\left(\tilde{y}_{t}\right)\right\} d \tau \\
& -\frac{\delta\left(R^{*}\right)^{2}}{\gamma S^{*}}(\mu+\delta)\left(z_{t}-1\right)^{2} .
\end{aligned}
$$

By the condition (1.5) and Lemma 4.1, we obtain

$$
\begin{aligned}
\frac{d U_{\delta}^{E^{*}}(t)}{d t}= & -\left(\mu_{1} S^{*}+\delta\left(R(t)-R^{*}\right)\right) \frac{\left(x_{t}-1\right)^{2}}{x_{t}}-\frac{\delta}{S^{*}}\left\{R^{*}\left(z_{t}-1\right)-\frac{N^{*}}{2}\left(n_{t}-1\right)\right\}^{2} \\
& -\int_{0}^{h} f(\tau)\left\{g\left(\frac{1}{x_{t}}\right)+g\left(\frac{x_{t} \tilde{y}_{t, \tau}}{y_{t}}\right)+g\left(y_{t}\right)-g\left(\tilde{y}_{t}\right)\right\} d \tau \\
& -\frac{\delta\left(R^{*}\right)^{2}}{\gamma S^{*}}(\mu+\delta)\left(z_{t}-1\right)^{2} \\
\leq & -\frac{\delta}{S^{*}}\left\{R^{*}\left(z_{t}-1\right)-\frac{N^{*}}{2}\left(n_{t}-1\right)\right\}^{2}-\int_{0}^{h} f(\tau)\left\{g\left(\frac{1}{x_{t}}\right)+g\left(\frac{x_{t} \tilde{y}_{t, \tau}}{y_{t}}\right)\right\} d \tau \\
& -\frac{\delta\left(R^{*}\right)^{2}}{\gamma S^{*}}(\mu+\delta)\left(z_{t}-1\right)^{2} .
\end{aligned}
$$


From (4.8) and (4.10), for the both cases, we obtain $\frac{d U^{E^{*}}(t)}{d t} \leq 0$ for all $t>0$ with equality if and only if $S(t)=S^{*}, R(t)=R^{*}$. This implies $\lim _{t \rightarrow+\infty} S(t)=S^{*}$, $\lim _{t \rightarrow+\infty} R(t)=R^{*}$, that is $\lim _{t \rightarrow+\infty} I(t)=I^{*}$ holds. By an extension of LaSalle invariance principle (see also Kuang [11, Corollary 5.2]), the endemic equilibrium $E^{*}$ is globally asymptotically stable. This completes the proof.

Proof of Theorem 1.2. We consider the following Lyapunov functional.

$$
U_{\delta}^{E^{0}}(t)=\left\{\begin{array}{l}
U_{0}^{E^{0}}(t)+\frac{\delta}{\gamma S^{0}} \frac{\left(R(t)-R^{0}\right)^{2}}{2}+\frac{\delta \gamma}{\left\{\gamma\left(\mu_{3}-\mu_{1}\right)+\left(\mu_{2}-\mu_{1}\right)\left(\mu_{1}+\mu_{3}+\delta\right)\right\} S^{0}} \frac{\left\{\left(N(t)-N^{0}\right)+\frac{\mu_{2}-\mu_{1}}{\gamma}\left(R(t)-R^{0}\right)\right\}^{2}}{2}, \\
\text { if either } \mu_{1}<\mu_{2} \text { or } \mu_{1}<\mu_{3} \\
U_{0}^{E^{0}}(t)+\frac{\delta}{\gamma S^{0}} \frac{\left(R(t)-R^{0}\right)^{2}}{2}+\frac{\delta}{4 \mu_{1} S^{0}} \frac{\left(N(t)-N^{0}\right)^{2}}{2} \\
\text { if } \mu_{1}=\mu_{2}=\mu_{3},
\end{array}\right.
$$

where $U_{0}^{E^{0}}(t)$ is defined in (3.2). First, the time derivative of $U_{0}^{E^{0}}(t)$ along the solution of system (1.2) becomes as follows.

$$
\frac{d U_{0}^{E^{0}}(t)}{d t}=-\mu_{1} \frac{\left(S(t)-S^{0}\right)^{2}}{S(t)}+\left(\mu_{2}+\gamma\right)\left(R_{0} \frac{G(I(t))}{I(t)}-1\right) I(t)+\delta\left(1-\frac{S^{0}}{S(t)}\right)\left(R(t)-R^{0}\right)
$$

Second, calculating the time derivatives of $\frac{\delta}{\gamma S^{0}} \frac{\left(R(t)-R^{0}\right)^{2}}{2}$ gives

$$
\begin{aligned}
& \frac{d}{d t}\left\{\frac{\delta}{\gamma S^{0}} \frac{\left(R(t)-R^{0}\right)^{2}}{2}\right\} \\
= & \frac{\delta}{\gamma S^{0}}\left(R(t)-R^{0}\right)\left\{\gamma I(t)-\left(\mu_{3}+\delta\right)\left(R(t)-R^{0}\right)\right\} \\
= & \frac{\delta}{\gamma S^{0}}\left(R(t)-R^{0}\right)\left\{\gamma(N(t)-S(t)-R(t))-\left(\mu_{3}+\delta\right)\left(R(t)-R^{0}\right)\right\} \\
= & \frac{\delta}{\gamma S^{0}}\left(R(t)-R^{0}\right)\left\{\gamma\left(N(t)-N^{0}\right)-\gamma\left(S(t)-S^{0}\right)-\left(\mu_{3}+\gamma+\delta\right)\left(R(t)-R^{0}\right)\right\} \\
= & \frac{\delta}{S^{0}} R(t)\left(N(t)-N^{0}\right)-\delta\left(R(t)-R^{0}\right)\left(\frac{S(t)}{S^{0}}-1\right)-\frac{\delta\left(\mu_{3}+\gamma+\delta\right)}{\gamma S^{0}}\left(R(t)-R^{0}\right)^{2} .
\end{aligned}
$$


For the first case either $\mu_{1}<\mu_{2}$ or $\mu_{1}<\mu_{3}$, similar to (4.6), we obtain

$$
\begin{aligned}
& \frac{d}{d t}\left\{\frac{\left.\left\{\left(N(t)-N^{0}\right)+\frac{\mu_{2}-\mu_{1}}{\gamma}\left(R(t)-R^{0}\right)\right\}^{2}\right\}}{2}\right\} \\
= & \left\{\left(N(t)-N^{0}\right)+\frac{\mu_{2}-\mu_{1}}{\gamma}\left(R(t)-R^{0}\right)\right\}\left\{B-\mu_{1} S(t)-\mu_{2} I(t)-\mu_{3} R(t)-\frac{\mu_{2}-\mu_{1}}{\gamma}\left(\gamma I(t)-\mu_{3} R(t)\right)\right\} \\
= & \left\{\left(N(t)-N^{0}\right)+\frac{\mu_{2}-\mu_{1}}{\gamma}\left(R(t)-R^{0}\right)\right\} \\
& \times\left\{B-\mu_{1}(N(t)-I(t)-R(t))-\mu_{2} I(t)-\mu_{3} R(t)-\frac{\mu_{2}-\mu_{1}}{\gamma}\left(\gamma I(t)-\mu_{3} R(t)\right)\right\} \\
= & \left\{\left(N(t)-N^{0}\right)+\frac{\mu_{2}-\mu_{1}}{\gamma}\left(R(t)-R^{0}\right)\right\} \\
& \times\left\{B-\mu_{1} N(t)-\left(\mu_{2}-\mu_{1}\right) I(t)-\left(\mu_{3}-\mu_{1}\right) R(t)-\frac{\mu_{2}-\mu_{1}}{\gamma}\left(\gamma I(t)-\left(\mu_{3}+\delta\right) R(t)\right)\right\} \\
= & \left\{\left(N(t)-N^{0}\right)+\frac{\mu_{2}-\mu_{1}}{\gamma}\left(R(t)-R^{0}\right)\right\}\left[B-\mu_{1} N(t)-\left\{\left(\mu_{3}-\mu_{1}\right)+\frac{\left(\mu_{2}-\mu_{1}\right)\left(\mu_{3}+\delta\right)}{\gamma} R(t)\right\}\right] \\
= & \left\{\left(N(t)-N^{0}\right)+\frac{\mu_{2}-\mu_{1}}{\gamma}\left(R(t)-R^{0}\right)\right\} \\
& \times\left[-\mu_{1}\left(N(t)-N^{0}\right)-\left\{\left(\mu_{3}-\mu_{1}\right)+\frac{\left(\mu_{2}-\mu_{1}\right)\left(\mu_{3}+\delta\right)}{\gamma}\right\}\left(R(t)-R^{0}\right)\right] \\
= & -\mu_{1}\left(N(t)-N^{0}\right)^{2}-\left\{\left(\mu_{3}-\mu_{1}\right)+\frac{\left(\mu_{2}-\mu_{1}\right)\left(\mu_{1}+\mu_{3}+\delta\right)}{\gamma}\right\}\left(N(t)-N^{0}\right)\left(R(t)-R^{0}\right) \\
& -\frac{\mu_{2}-\mu_{1}}{\gamma}\left\{\left(\mu_{3}-\mu_{1}\right)+\frac{\left(\mu_{2}-\mu_{1}\right)\left(\mu_{3}+\delta\right)}{\gamma}\right\}\left(R(t)-R^{0}\right)^{2} .
\end{aligned}
$$

Combining (4.11), (4.12) and (4.13), we have

$$
\begin{aligned}
& \frac{d U_{\delta}^{E^{0}}(t)}{d t} \\
= & -\mu_{1} \frac{\left(S(t)-S^{0}\right)^{2}}{S(t)}+\left(\mu_{2}+\gamma\right)\left(R_{0} \frac{G(I(t))}{I(t)}-1\right) I(t)+\delta\left(1-\frac{S^{0}}{S(t)}\right)\left(R(t)-R^{0}\right) \\
& +\frac{\delta}{S^{0}}\left(R(t)-R^{0}\right)\left(N(t)-N^{0}\right)-\delta\left(R(t)-R^{0}\right)\left(\frac{S(t)}{S^{0}}-1\right)-\frac{\delta\left(\mu_{3}+\gamma+\delta\right)}{\gamma S^{0}}\left(R(t)-R^{0}\right)^{2} \\
& -\frac{\mu_{1} \delta \gamma}{\left\{\gamma\left(\mu_{3}-\mu_{1}\right)+\left(\mu_{2}-\mu_{1}\right)\left(\mu_{1}+\mu_{3}+\delta\right)\right\} S^{0}}\left(N(t)-N^{0}\right)^{2}-\frac{\delta}{S^{0}}\left(N(t)-N^{0}\right)\left(R(t)-R^{0}\right) \\
& -\frac{\delta\left(\mu_{2}-\mu_{1}\right)\left\{\gamma\left(\mu_{3}-\mu_{1}\right)+\left(\mu_{2}-\mu_{1}\right)\left(\mu_{3}+\delta\right)\right\}}{\gamma\left\{\gamma\left(\mu_{3}-\mu_{1}\right)+\left(\mu_{2}-\mu_{1}\right)\left(\mu_{1}+\mu_{3}+\delta\right)\right\} S^{0}}\left(R(t)-R^{0}\right)^{2} .
\end{aligned}
$$


Similar to Lemma 4.1, we use the following equation (see [16]).

$$
\begin{aligned}
& \left(1-\frac{S^{0}}{S(t)}\right)\left(R(t)-R^{0}\right)-\left(R(t)-R^{0}\right)\left(\frac{S(t)}{S^{0}}-1\right) \\
= & \left(1-\frac{S^{0}}{S(t)}\right)\left(1-\frac{S(t)}{S^{0}}\right)\left(R(t)-R^{0}\right) \\
= & -\frac{\left(S(t)-S^{0}\right)^{2}}{S^{0} S(t)} R(t) \leq 0 .
\end{aligned}
$$

Then, by (4.14), we obtain

$$
\begin{aligned}
\frac{d U_{\delta}^{E^{0}}(t)}{d t}= & -\left(\mu_{1} S^{0}+\delta R(t)\right) \frac{\left(S(t)-S^{0}\right)^{2}}{S^{0} S(t)}+\left(\mu_{2}+\gamma\right)\left(R_{0} \frac{G(I(t))}{I(t)}-1\right) I(t) \\
& -\frac{\mu_{1} \delta \gamma}{\left\{\gamma\left(\mu_{3}-\mu_{1}\right)+\left(\mu_{2}-\mu_{1}\right)\left(\mu_{1}+\mu_{3}+\delta\right)\right\} S^{*}}\left(N(t)-N^{0}\right)^{2} \\
& -\frac{\delta\left(\mu_{2}-\mu_{1}\right)\left\{\gamma\left(\mu_{3}-\mu_{1}\right)+\left(\mu_{2}-\mu_{1}\right)\left(\mu_{3}+\delta\right)\right\}}{\gamma\left\{\gamma\left(\mu_{3}-\mu_{1}\right)+\left(\mu_{2}-\mu_{1}\right)\left(\mu_{1}+\mu_{3}+\delta\right)\right\} S^{0}}\left(R(t)-R^{0}\right)^{2} \\
\leq & -\left(\mu_{1} S^{0}+\delta R(t)\right) \frac{\left(S(t)-S^{0}\right)^{2}}{S^{0} S(t)}+\left(\mu_{2}+\gamma\right)\left(R_{0}-1\right) I(t) \\
& -\frac{\mu_{1} \delta \gamma}{\left\{\gamma\left(\mu_{3}-\mu_{1}\right)+\left(\mu_{2}-\mu_{1}\right)\left(\mu_{1}+\mu_{3}+\delta\right)\right\} S^{*}}\left(N(t)-N^{0}\right)^{2} \\
& -\frac{\delta\left(\mu_{2}-\mu_{1}\right)\left\{\gamma\left(\mu_{3}-\mu_{1}\right)+\left(\mu_{2}-\mu_{1}\right)\left(\mu_{3}+\delta\right)\right\}}{\gamma\left\{\gamma\left(\mu_{3}-\mu_{1}\right)+\left(\mu_{2}-\mu_{1}\right)\left(\mu_{1}+\mu_{3}+\delta\right)\right\} S^{0}}\left(R(t)-R^{0}\right)^{2} .
\end{aligned}
$$

For the second case $\mu_{1}=\mu_{2}=\mu_{3}$, similar to (4.9), we obtain

$$
\frac{d}{d t}\left\{\frac{\delta}{4 \mu_{1} S^{0}} \frac{\left(N(t)-N^{0}\right)^{2}}{2}\right\}=-\frac{\delta}{4 S^{0}}\left(N(t)-N^{0}\right)^{2} .
$$

By (4.14), we obtain

$$
\begin{aligned}
\frac{d U_{\delta}^{E^{0}}(t)}{d t}= & -\left(\mu_{1} S^{0}+\delta R(t)\right) \frac{\left(S(t)-S^{0}\right)^{2}}{S^{0} S(t)}+\left(\mu_{2}+\gamma\right)\left(R_{0} \frac{G(I(t))}{I(t)}-1\right) I(t) \\
& -\frac{\delta}{S_{0}} R(t)^{2}+\frac{\delta}{S_{0}} R(t)\left(N(t)-N^{0}\right)-\frac{\delta}{4 S_{0}}\left(N(t)-N^{0}\right)^{2}-\frac{\delta\left(\mu_{3}+\delta\right)}{\gamma S_{0}} R(t)^{2} \\
\leq & -\left(\mu_{1} S^{0}+\delta R(t)\right) \frac{\left(S(t)-S^{0}\right)^{2}}{S^{0} S(t)}+\left(\mu_{2}+\gamma\right)\left(R_{0}-1\right) I(t) \\
& -\frac{\delta}{S_{0}}\left\{R(t)-\frac{\left(N(t)-N^{0}\right)}{2}\right\}^{2}-\frac{\delta\left(\mu_{3}+\delta\right)}{\gamma S_{0}} R(t)^{2}
\end{aligned}
$$

From (4.15) and (4.16), for the both cases, we obtain $\frac{d U_{\delta}^{E^{0}}(t)}{d t} \leq 0$ for all $t>0$ with equality if and only if $S(t)=S^{0}, R(t)=R^{0}$ and $N(t)=N^{0}$. Therefore, we have $\lim _{t \rightarrow+\infty} S(t)=S^{0}, \lim _{t \rightarrow+\infty} R(t)=R^{0}$ and $\lim _{t \rightarrow+\infty} N(t)=N^{0}$, which imply that $\lim _{t \rightarrow+\infty} I(t)=I^{0}$ holds. By an extension of LaSalle invariance principle (see also Kuang [11, Corollary 5.2]), the disease-free equilibrium $E^{0}$ is globally asymptotically stable. From Lemma 4.2 , the proof is complete. 


\section{Conclusion}

To investigate global behavior of disease prevalence has played a vital role to predict the dynamics of the disease transmission in the long run and take more efficient control measures such as vaccination for immunization in the communicable diseases.

In this paper, by applying deformation techniques of the time deriavtive of Lyapunov functionals in Nakata et al. [16] (see Lemma 4.1) and constructing a Lyapunov functional $U_{\delta}^{E^{0}}$ (resp. $U_{\delta}^{E^{*}}$ ) for $R_{0} \leq 1$ (resp. $R_{0}>1$ ), we established the global asymptotic stability of the disease-free equilibrium $E^{0}$ (resp. the endemic equilibrium $E^{*}$ ) of an SIRS epidemic model with a class of nonlinear incidence rates and distributed delays for $R_{0} \leq 1$ (resp. $R_{0}>1$ ).

Our model incorporates the assumption that the death rates of susceptible, infective and recovered individuals is different each other and the monotone properties of $G(I)$ and $I / G(I)$ in (H1) and (H2) are satisfied when considering a class of nonlinear incidence rates which describes saturation effects observed in the literature of epidemiology [3]. Theorems 1.1 and 1.2 show that, if $R_{0} \leq$ 1 , then the diseases transmission with impermanent immunity will eventually disappear, and if $R_{0}>1$, then the diseases will be permanent. Furthermore, without imposing any restriction on the size of a latent period $h$, if the basic reproduction number $R_{0}$ lies in an interval $\left(1,1+\mu_{2} / \gamma\right]$, then the disease will equilibrate at an endemic steady state for any rate of immunity loss $\delta$ and otherwise, we establish the maximal rate of immunity loss $\bar{\delta}$ which guarantees the global stability of the endemic steady state.

\section{Acknowledgements}

The authors wish to express their gratitude to the editor and an anonymous referee for the helpful comments and valuable suggestion which improved the quality of this paper. The authors' work was supported in part by JSPS Fellows, No.237213 of Japan Society for the Promotion of Science to the first author, by the Grant MTM2010-18318 of the MICINN, Spanish Ministry of Science and Innovation to the second author, and by Scientific Research (c), No.21540230 of Japan Society for the Promotion of Science to the third author. The work of this paper was partially prepared at Technische Universität Darmstadt from the beginning of October to the end of November 2011 participating in the International Research Training Group Mathematical Fluid Dynamics funded by Deutsche Forschungsgemeinschaft (DFG) and Japan Society for the Promotion of Science (JSPS).

\section{References}

[1] E. Beretta, Y. Takeuchi, Convergence results in SIR epidemic models with varying population size, Nonlinear Anal. 28 (1997) 1909-1921. 
[2] E. Beretta, T. Hara, W. Ma, Y. Takeuchi, Global asymptotic stability of an SIR epidemic model with distributed time delay, Nonlinear Anal. 47 (2001) 4107-4115.

[3] V. Capasso and G. Serio, A generalization of the Kermack-Mckendrick deterministic epidemic model, Math. Biosci. 42 (1978) 43-61.

[4] J. Chen, An SIRS epidemic model, Appl. Math. J. Chinese Univ. 19 (2004) 101-108.

[5] Y. Enatsu, Y. Nakata and Y. Muroya, Global stability for a class of discrete SIR epidemic models, Math. Biosci. Engi. 2 (2010) 347-361.

[6] Y. Enatsu, Y. Nakata and Y. Muroya, Global stability of SIR epidemic models with a wide class of nonlinear incidence rates and distributed delays, Dis. Cont. Dynam. Sys. B 15 (2011) 61-74.

[7] Y. Enatsu, E. Messina, Y. Nakata, Y. Muroya, E. Russo and A. Vecchio, Global dynamics of a delayed SIRS epidemic model with a wide class of nonlinear incidence rates, J. Appl. Math. Comp. doi:10.1007/s12190-011$0507-\mathrm{y}$.

[8] G. Huang and Y. Takeuchi, Global analysis on delay epidemiological dynamics models with nonlinear incidence, J. Math. Biol. 63 (2011) 125-139.

[9] G. Huang, Y. Takeuchi, W. Ma and D. Wei, Global stability for delay SIR and SEIR epidemic models with nonlinear incidence rate, Bull. Math. Biol. 72 (2010) 1192-1207.

[10] A. Korobeinikov, Global Properties of Infectious Disease Models with Nonlinear Incidence, Bull. Math. Biol. 69 (2007) 1871-1886.

[11] Y. Kuang, Delay Differential Equations with Applications in Population Dynamics. Academic Press, San Diego, 1993.

[12] C. C. McCluskey, Complete global stability for an SIR epidemic model with delay-Distributed or discrete, Nonl. Anal. RWA. 11 (2010) 55-59.

[13] C. C. McCluskey, Global stability for an SIR epidemic model with delay and nonlinear incidence, Nonl. Anal. RWA. 11 (2010) 3106-3109.

[14] C. C. McCluskey, Global stability of an SIR epidemic model with delay and general nonlinear incidence, Math. Biosci. Engi. 7 (2010) 837-850.

[15] J. Mena-Lorcat and H.W. Hethcote, Dynamic models of infectious diseases as regulators of population sizes, J. Math. Biol. 30 7(1992) 693-716.

[16] Y. Nakata, Y. Enatsu and Y. Muroya, On the global stability of an SIRS epidemic model with distributed delays, accepted. 
[17] Y. Takeuchi, W. Ma and E. Beretta, Global asymptotic properties of a delay SIR epidemic model with finite incubation times, Nonlinear Anal. 42 (2000) 931-947.

[18] W. Wang, Global behavior of an SEIRS epidemic model with time delays, Appl. Math. Lett. 15 (2002) 423-428.

[19] D. Xiao and S. Ruan, Global analysis of an epidemic model with nonmonotone incidence rate, Math. Biosci. 208 (2007) 419-429.

[20] R. Xu and Z. Ma, Stability of a delayed SIRS epidemic model with a nonlinear incidence rate, Chaos, Solitons and Fractals. 41 (2009) 23192325 .

[21] Y. Yang and D. Xiao, Influence of latent period and nonlinear incidence rate on the dynamics of SIRS epidemiological models, Dis. Cont. Dyn. Sys. Ser. B, 13 (2010) 195-211.

[22] T. Zhang and Z. Teng, Global behavior and permanence of SIRS epidemic model with time delay, Nonl. Anal. RWA. 9 (2008) 1409-1424.

[23] J. Zhen, Z. Ma and M. Han, Global stability of an SIRS epidemic model with delays, Acta. Math. Sci. 26B (2006) 291-306. 